\title{
Synchronous Male Breast Cancer and a Review of the
}

\section{Literature}

\author{
Liu $\mathrm{Z}^{1 *}$, Shaffer $\mathrm{K}^{2}$ and Hines $\mathrm{N}^{2}$ \\ 1Brown University Warren Alpert medical school/Rhode Island Hospital, USA \\ ${ }^{2}$ Boston University Medical Center, USA
}

*Corresponding author: Z Liu, Brown University Warren Alpert Medical

\section{Case Report}

Volume 3 Issue 2

Received Date: February 21, 2019

Published Date: March 25, 2019

School/Rhode Island Hospital, 593 Eddy Street, Providence RI 02903, USA, Tel: 3149222964; Email: zliu00@gmail.com

\section{Abstract}

The incidence of male breast cancer is uncommon, occurring in $<1 \%$ of the population. Synchronous breast cancer in men is exceedingly rare and less common than metachronous bilateral breast cancer. One study spanning 20 years and involving 2524 male breast cancer patients estimated that $2.1 \%$ were synchronous while $2.3 \%$ were metachronus. We present a case of synchronous breast cancer in a 70 year-old male with a family history of breast cancer. In this case report we review the epidemiology, radiologic workup, imaging findings and management decisions for male breast cancer.

Keywords: Breast Cancer; Male Breast Cancer; Synchronous Male Breast Cancer

Abbreviations: ER: Estrogen Receptor; PR: Progresterone Receptor; HER2: Human Epidermal Growth Factor Receptor 2.

\section{Introduction}

Male breast cancer is uncommon, occurring in less than $1 \%$ of all cases of breast cancer [1-8]. Synchronous bilateral breast cancer is rare and less common than metachronous bilateral breast cancer [1,3-9]. In a cohort of 2524 male breast cancer patients diagnosed between 1988 and 2008, the incidence of synchronous male breast cancer was estimated to be $2.1 \%$, while $2.3 \%$ were metachronus [9]. In a cohort of 123,757 female breast cancer patients diagnosed between 1970 and 2000, the incidence of synchronous female breast cancer was estimated to be $1.6 \%$, while $3.8 \%$ were metachronous [10]. Most cases of male breast cancer are detected between the age of 60 to 70 , and the mean age is 67 years [1,3-8]. Due to lack of routine breast cancer screening, most male breast cancers are diagnosed at a later stage
(Stage II in men versus Stage I in women) and therefore conferring a lower survival rate [11]. Most of male breast cancers are invasive ductal carcinomas [11]. In this case report we will review the radiologic workup of men presenting with breast complaints, imaging findings, epidemiology and management of male breast cancer.

\section{Case Report}

A 70 year-old man presented to his primary care physician with a $1 \mathrm{~cm}$ palpable lump in his right breast for 2 months. He did not complain of pain, skin changes or discharge. The patient denied history of trauma. Of note, the patient reported a family history of breast cancer in his mother and 2 sisters. No other risk factors for male breast cancer were present. These risk factors include radiation exposure, endocrine abnormalities, obesity or testicular disorders (cryptochidism, Mumps orchitis, orchiectomy) or Klinefelter's syndrome [2-8].

Physical exam noted the right breast was asymmetrically enlarged with a mobile, irregular mass in 


\section{Clinical Radiology \& Imaging Journal}

the 12-1 o'clock position, $1 \mathrm{~cm}$ from the nipple. Mild tenderness was present with palpation. The left breast demonstrated subareolar fullness without a discrete mass or pain. No skin changes or discharge was noted. The axillae were clinically normal. Bilateral diagnostic mammogram was performed. A lobulated mass with irregular margins measuring $11 \times 16 \mathrm{~mm}$ was present at the 12:00 position of the right breast, $1 \mathrm{~cm}$ from the nipple (Figure 1). A smaller gently lobulated mass with irregular margins was seen in the subareolar left breast measuring 9 x $5 \mathrm{~mm}$ (Figure 2). There were no suspicious calcifications or architectural distortion associated with either mass.

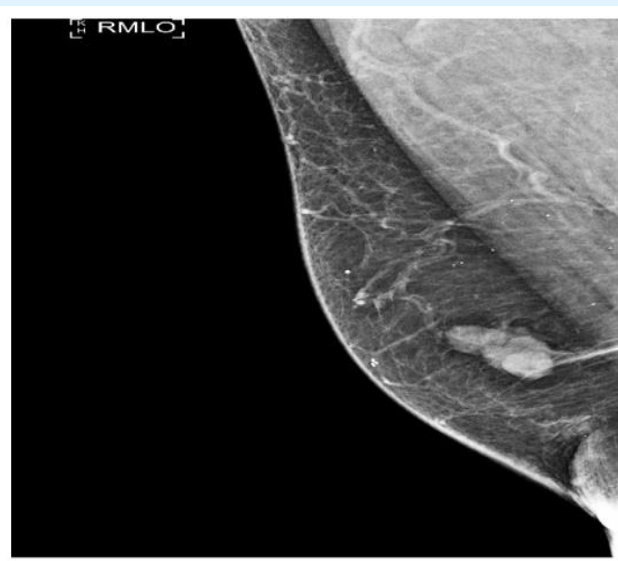

Figure 1: MLO view of right breast demonstrates an irregular high-density mass with microlobuated margins eccentric to the nipple.

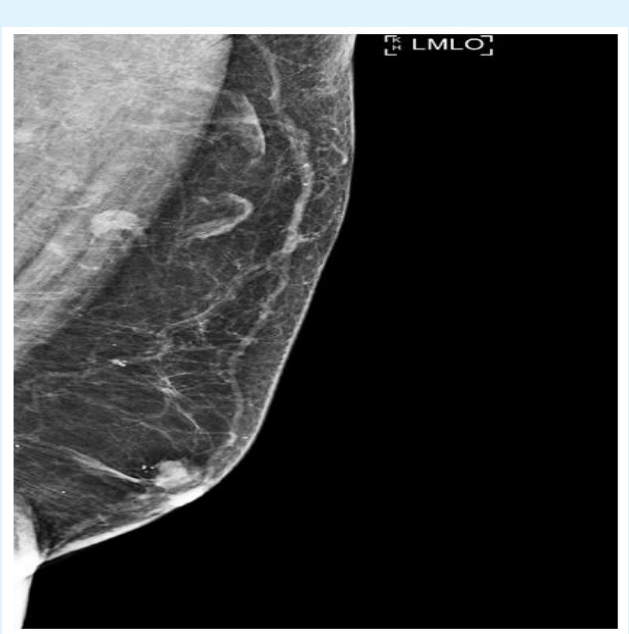

Figure 2: MLO view of left breast demonstrates an irregular high-density mass with microlobuated margins in a subareolar location. Normal appearing lymph nodes are seen in the axilla.
Sonographic evaluation of the right breast at the $12 \mathrm{o}^{\prime}$ clock, subareolar position demonstrated an irregular hypoechoic mass measuring 14 x 14 x $14 \mathrm{~mm}$ (Figure 3). Sonographic evaluation of the left breast demonstrated an irregular hypoechoic subareolar mass measuring $10 \times 10$ x $7 \mathrm{~mm}$ (Figure 4). Morphologically normal lymph nodes were seen in both axillas. Ultrasound guided core biopsy was performed of both masses.
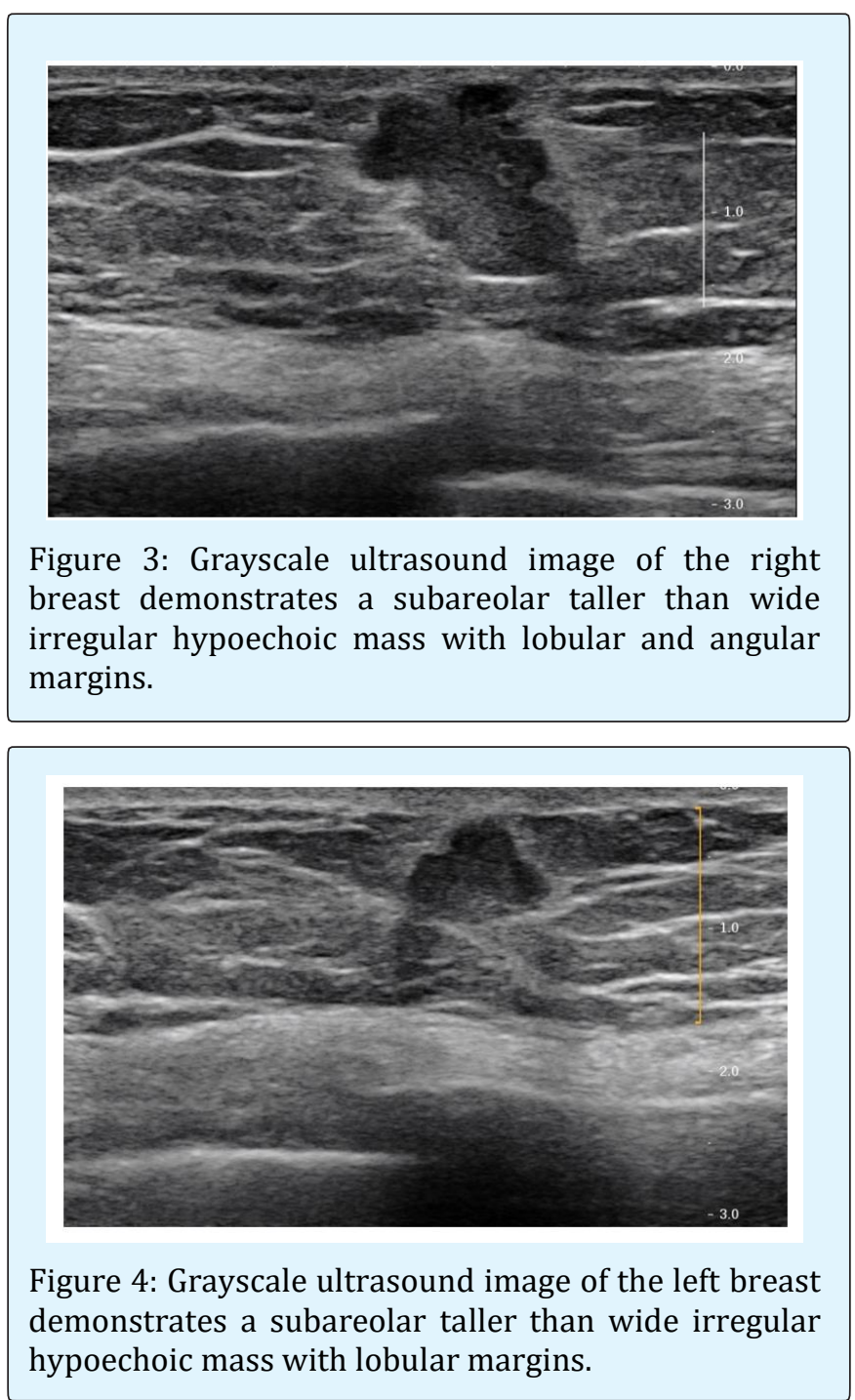

Histopathology studies showed bilateral invasive ductal carcinoma, grade II/III using modified ScarffBloom-Richardson grading system. No lymphovascular space invasion, microcalcifications, or intraductal components were present in either tumor. The tumor in the right breast demonstrated strong estrogen receptor (ER) positivity ( $99 \%$ of tumor nuclei) and progresterone receptor (PR) positivity (97\% of tumor nuclei). The 


\section{Clinical Radiology \& Imaging Journal}

immunoreactivity for MIB-1/Ki-67 monoclonal antibody revealed $18 \%$ positive staining (intermediate proliferation rate). HER2/neu was negative. The tumor in the left breast demonstrated strong ER positivity (99\%) and PR positivity (98\%). The MIB-1/Ki-67 monoclonal antibody revealed $24 \%$ tumor staining (intermediate proliferation rate). HER2/neu was negative.

\section{Discussion}

Breast cancer in men is rare, accounting for approximately $1 \%$ of all breast cancer cases in the United States and $0.1 \%$ of cancer mortality in men [1]. In contrast to female breast cancer, male breast cancers tend to occur later in life andpresent at a later stage. The median age at diagnosis is 67 years for men compared with 62 years for women [11]. Risk factors include BRCA gene mutation, family history of breast or ovarian cancer, personal history of cancer (prostate, pancreatic and testicular cancers), and history of radiation treatment to the chest, Klinefelter's (XXY) syndrome, and obesity [2-8]. The rate of male breast cancer has increased over the last several decades $[2,11]$. In 1975, the incidence of male breast cancer was 1.0 case per 100,000. In 2010 the incidence was 1.2 cases per 100,000 men. The increase has been limited to in situ and localized tumors, which may be secondary to increased awareness and follow up of breast symptoms $[2,11,12]$. Unlike female breast cancer, incidence rates are higher in African American men than Caucasian men $[2,12]$. Due to lack of routine breast cancer screening and possibly lack of public awareness, men often present with higher stage disease, larger tumors and more frequent lymph node involvement $[11,13]$.

The most common histologic subtype is invasive ductal carcinoma, representing 90\% [11,13]. Lobular carcinoma accounts for $1.5 \%$ of cases. This ratio is due to the lack of acini and lobules in normal male breast. However, lobules and acini may be present in patients taking exogenous estrogen [13]. The tumors are low grade and frequently demonstrate positive estrogen and progesterone receptor staining [11,12]. Overall, the current treatment for men diagnosed with breast cancer is similar to those guidelines currently used for postmenopausal women [12]. There are no guidelines for breast cancer screening in men. Diagnostic imaging is indicated for a male patient presenting with a breast lump or enlargement. The American College of Radiology (ACR) appropriateness criteria recommends ultrasound in men less than 25 years of age and diagnostic mammogram and ultrasound in men over 25 years of age [14].
The most common presentation for male breast cancer is a painless palpable mass $[11,15]$. Skin thickening and nipple retraction may be present. There is nipple involvement in $40-50 \%$ of cases. Gynecomastia or bloody nipple discharge is present in $16 \%$ of cases [15]. Mammography should be the initial modality for evaluation. Targeted ultrasound should only be obtained if there are suspicious features [16]. The mammographic appearance is a high-density irregular mass [17]. An eccentric subareolar location of the mass should raise suspicion for malignancy, differentiating it from gynecomastia $[17,18]$. Presence of microcalcifications is less commonly seen in males than in females. Nipple retraction, skin thickening, and increased trabeculation are secondary signs. The differential diagnosis includes gynecomastia, intraductal papilloma and papillary carcinoma $[17,19,20]$.

The most common differential diagnosis for male breast cancer is gynecomastia, the most common abnormality of the male breast. Gynecomastia is caused by benign proliferation of the subareolar ducts and the surrounding stroma due to endogenous hormone instability [16-18]. There is a bimodal distribution, first seen at puberty, with a second peak around age 50 [1618]. Underlying causes include exogenous hormones, hormone producing tumors, liver or renal disease and hyperthyroidism [18]. Drugs such as cimetidine, thiazides, spironolactone as well as marajuana have been linked to development of gynecomastia [15]. There is no strong evidence to suggest that gynecomastia increases risk of breast cancer [16-18,21].

Imaging appearance of gynecomastia on mammography ranges from a nodular, dendritic or diffuse glandular pattern centered at the nipple [16]. The nodular pattern appears as a fan-shaped subareolar density that blends with the surrounding fat. The dentritic or chronic fibrotic phase is characteriszed by "flame-shaped" subareolar density radiating from the nipple that may extend into the upper outer quadrant. Diffuse glandular pattern can be seen in patients receiving high-dose estrogen therapy. Gynecomastia is most often asymmetric and bilateral; however, it can present unilaterally or bilateral and symmetric [16-18,21,22]. If classic gynecomastia is seen on mammogram with no microcalcifications or eccentric mass, ultrasound is not indicated. On ultrasound, the nodular pattern typically shows a subareolar hypoechoic mass with lobulation or even spiculation correponding to the palpable mass [20]. Sonographic findings can appear malignant and therefore, mammographic analysis is crucial for differentiation between benign and malignant disease. Clinically, 


\section{Clinical Radiology \& Imaging Journal}

gynecomastia manifests as a concentric, mobile soft subareolar mass that may be painful, which is not a common finding in cancer.

In contrast, pseudogynecomastia most commonly presents in patients that are obese. Pseudogynecomastia manifests clinically as unilateral or bilateral breast enlargement rather than a discrete mass. Pseudogynecomastia is caused by proliferation of normal fatty tissue. There is no tissue density seen on mammography. A much less common differential is intraductal papilloma, which can present as a welldefined eccentric subareolar mass on mammogram [20]. It is characterized by benign proliferation of the intraductal mammary epithelium and there are two broad types: central and peripheral with the central type being solitary and subareolar in location within a major duct. Appearance on ultrasound is typically a well-defined solid nodule or intraductal mass within a dilated duct. Conversely, it may appear as a hypoechoic mass associated with a cyst, with the cystic component representing anectatic duct $[18,20]$. Color Doppler may demonstrate a vascular stalk. Intraductal papilloma may be associated with gynecomastia. In situations where imaging findings are suggestive of an intraductal papilloma, further evaluation with tissue sampling and surgical excision is recommended because of potential malignant transformation [23]. Papillary carcinoma is also more common in men (3\% of male breast cancer) than women (1\%) [11,20]. Most male papillary carcinomas are intracystic and noninvasive. On mammogram, it appears as a round or oval mass. Focal poorly defined borders may also be seen, suggesting an invasive component. Sonographic appearance includes a solid mass or a complex cystic mass with thick walls containing both solid and cystic components.

\section{Summary}

Synchronous bilateral breast cancer in men occurs in $<1 \%$ of all male breast cancers. Multiple known risk factors (hereditary and non-hereditary) have been identified. The most common hereditary factors in men include BRCA2 mutation and non-BRCA familial mutations. Prognostication and management guidelines have been extrapolated from studies based on female breast cancer. The salient points here are that it is important to examine/image both breasts in men with one palpable lump and it is important for those with strong family history to obtain routine clinical breast exams. This case report raises the question that perhaps BRCA genetic testing should be indicated for men with strong family history.

\section{References}

1. Weiss JR, Moysich KB, Swede H (2005) Epidemiology of male breast cancer. Cancer Epidemiol Biomarkers Prev 14(1): 20-26.

2. American Cancer Society. Breast Cancer Facts \& Figures 2013-2014.

3. Kahla PB, Cassaro S, Vladimir FG, Wayne MG, Cammarata A (2005) Bilateral synchronous breast cancer in a male. Mt Sinai J Med 72(2): 120-123.

4. Nwashilli NJ, Ugiagbe EE (2015) Bilateral synchronous male breast cancer. Saudi Med J 36(3): 359-362.

5. Jagtap SV, Chougule PG, Khatib W, Shukla DB, Jagtap SS (2014) Male breast cancer: presenting as synchronous, large, bilateral masses. J Clin Diagn Res 8(4): 07-08.

6. Sosnovskikh I, Naninato P, Gatti G, Caldarella P, Masullo M, et al. (2007) Synchronous bilateral breast cancer in men: a case report and review of the literature. Tumori 93(2): 225-227.

7. Kappikeri VK, KriplaniAM (2015) Bilateral synchronous carcinoma breast- a rare case presentation. Springerplus 4: 193.

8. Sun WY, Lee KH, Lee HC, Ryu DH, Park JW, et al. (2012) Synchronous bilateral male breast cancer: a case report. J Breast Cancer 15(2): 248-251.

9. Kheirelseid EA, Jumustafa H, Miller N, Curran C, Sweeney K, et al. (2011) Bilateral breast cancer: analysis of incidence, outcome, survival and disease characteristics. Breast Cancer Res Treat 126(1): 131140.

10. Hartman M, Czene K, Reilly M, Adolfsson J, Bergh J, et al. (2007) Incidence and Prognosis of Synchronous and Metachronous Bilateral Breast Cancer. J Clin Oncol 25(27): 4210-4216.

11. Giordano SH, Cohen DS, Buzdar AU, Perkins G, Hortobagyi GN (2004) Breast carcinoma in men. Cancer 101(1): 51-57.

12. Harlan LC, Zujewski JA, Goodman MT, Stevens JL (2010) Breast Cancer in Men in the US: A populationbased study of diagnosis, treatment and survival. Cancer 116(15): 3558-3568. 


\section{Clinical Radiology \& Imaging Journal}

13. Vetto JT (2010) Breast diseases in males. In: Ismail Jatoi and Manfred Kaufmann (eds.), Management of Breast Diseases, Springer: 473-498.

14. Mainiero $\mathrm{MB}$, Lourenco AP, Barke LD, Argus AD, Bailey L, et al. (2015) ACR Appropriateness Criteria Evaluation of the Symptomatic Male Breast. J Am Coll Radiol 12(7): 678-682.

15. Aggarwal A, Liu ML, Krasnow SH (2014) Breast cancer in male veteran population: an analysis from VA cancer registry. J Community Support Oncol 12(8): 293-297.

16. Nguyen C, Kettler MD, Swirsky ME, Miller VI, Scott C, et al. (2013) Male Breast Disease: Pictorial Review with Radiologic-Pathologic Correlation. Radiographics 33(3): 763-779.

17. Chen L, Chantra PK, Larsen LH, Barton $P$, Rohitopakarn M, et al. (2006) Imaging characteristics of malignant lesions of the male breast. Radiographics 26(4): 993-1006.

18. Kopans DB (2007) Breast imaging. $3^{\text {rd }}$ (Edn.), Lipimncot Wms \& Wilkins, Philadelphia.
19. Honrado E, Benítez J, Palacios J (2005) The molecular pathology of hereditary breast cancer: genetic testing and therapeutic implications. Mod Pathol 18(10): 1305-1320.

20. Yitta S, Singer CI, Toth HB, Mercado CL (2010) Sonographic Appearances of Benign and Malignant Male Breast Disease with Mammographic and Pathologic Correlation. J Ultrasound Med 29(6): 931947.

21. Braunstein GD (2007) Clinical practice: gynecomastia. N Engl J Med 357(12): 1229-1237.

22. Cardenosa MD (2008) Breast imaging companion. $3^{\text {rd }}$ (Edn.), Lippincott Williams \& Wilkins, Philadelphia.

23. Sarakbi WA, Worku D, Escobar PF, Mokbel K (2006) Breast papillomas: current management with a focus on a new diagnostic and therapeutic modality. Int Semin Surg Oncol 3: 1. 